\title{
Strengthening publishing capacity to support academic medicine in South Africa
}

That the results of medical and health research must be disseminated is one of the fundamental principles of biomedical ethics. Enshrined in the World Medical Association's 1964 Declaration of Helsinki, ${ }^{[1]}$ the requirement for publication of findings, whether positive or negative, aims to ensure that the risk accepted by participants in research studies is balanced by the benefit of knowing their data helped to advance scientific knowledge.

But what happens if there are no journals interested in publishing the work? What if editors judge it as uninteresting? Or reviewers point out similar work has been published elsewhere? It may be deemed too 'specialist' for a general journal. Or too context specific to make the grade on the international stage.

For all South African (SA) researchers who have written papers that found no home, these scenarios should strike a resounding chord. Pressure to write more and more has been mounting since subsidies based on numbers of papers published in accredited journals were introduced by the Department of Higher Education and Training in 2004. The incentive worked almost immediately: a $50-60 \%$ increase in papers published between 2004 and 2008, compared with a decade earlier. ${ }^{[2]}$ Additional financial incentives for universities to encourage all doctors-in-training to do at least some research (MMed) have further swollen the submissions pool. But capacity in academic publishing, and particularly in peer review, has not increased at nearly the same rate. The result is a mismatch between the number of authors seeking publication in high-quality journals and the capacity of journals to respond.

Although a 2006 analysis of SA's academic publishing industry concluded that the country had an 'extraordinarily high' number of scholarly journals compared with the number of publishing scholars, the prominence of SA journals in internationally recognised indexes was found to be low, and quality among non-indexed journals was a problem. ${ }^{[3]}$ The report, published by the South African Academy of Sciences, further identified a 'significantly ageing cohort of actively publishing scientists in the SA science system', meaning that younger authors are not getting the support they need to establish their publishing careers, and are not joining their senior colleagues in the ranks of journal editorial boards or reviewer networks.

This context led us at HMPG to reflect on the role of journals in SA's scientific development, and what we should be doing to help. Our conclusion was that we can - and should - be far more systematic and strategic in our work to ensure that we actively recruit wider networks of reviewers and academic editors, contribute to sharing knowledge about publishing processes, and support early- to mid-career professionals to become involved in peer review on a routine basis.

To this end, we have established two clear goals for our publishing activities: (i) to support the advancement of academic medicine in
SA by promoting the publication and dissemination of all health and medical research that meets quality standards; and (ii) to contribute to the strengthening of SA's research culture by anchoring our peer review and editorial processes within the academic communities we intend to support.

In order to implement the necessary changes to our internal process and external relationships, we have refocused our work around the priorities of researchers: fast turnaround times for peer review, publication as soon as possible after acceptance, wide distribution of published content through international online platforms (PubMed, Sabinet, Scopus, SciELO, DOAJ, among others), and provision of more extensive administrative support to authors, reviewers and academic editors. Coupled with these changes, we have made the decision to be far more inclusive in our editorial policy - with the support of an expanded team of associate editors drawn from academia and with expertise spanning the spectrum of health and medical research. We have revised our guidelines to authors to reflect the fact that we are actively seeking submissions from researchers in all health and medical disciplines and have launched a new highend online submission system to make the process of submission as smooth as possible. All these changes are aimed at ensuring that the $S A M J$ becomes the first-choice journal for publication of health and medical research with relevance to the SA context, and fulfils its aim to act as a platform for the exchange of crucial new knowledge among the country's academic medical communities.

We acknowledge that one journal cannot be 'all things to all people' but hope that by setting out a clear intention to respond to the needs of researchers in SA, and help address the capacity gaps in academic publishing, we will forge the strong relationships we need to make sure we stay on the right track.

\section{Hannah Kikaya \\ CEO and publisher \\ Health and Medical Publishing Group}

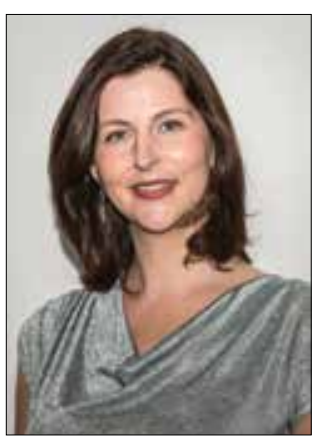

1. World Medical Association. Declaration of Helsinki - Ethical Principles for Medical Research Involving Human Subjects. Helsinki: WMA, 1964. http://www.wma.net/en/30publications/10policies/ b3/ (accessed 16 November 2016).

Woodiwiss AJ. Publication subsidies: Challenges and dilemmas facing South African researchers. Cardiovasc J Afr 2012;23(8):421-427.

3. Gevers W, Hammes M, Mati X, et al. Report on a strategic approach to research publishing in South Africa. Pretoria: Academy of Sciences of South Africa, 2006. http://www.assaf.org.za/files/2011/02/2466ASSAF-Strategic-approach-to-research-publishing-2.pdf (accessed 16 November 2016).

S Afr Med J 2016;106(12):1148. DOI:10.7196/SAMJ.2016.v106i12.12202 